\title{
Lung Ultrasound in COVID 19 Outbreak: Can we Reduce the Burden from the Overloaded CT Departments?
}

\author{
Filipe Penna de Carvalho ${ }^{1,2}$ and Alex dos Santos Felix ${ }^{1,2,3,4}$ \\ Américas Serviços Médicos, ${ }^{1}$ Rio de Janeiro, RJ - Brazil. \\ Diagnósticos da America SA (DASA), ${ }^{2}$ Rio de Janeiro, RJ - Brazil. \\ Clínica São Vicente, ${ }^{3}$ Rio de Janeiro, $R J$ - Brazil. \\ Instituto Nacional de Cardiologia, ${ }^{4}$ Rio de Janeiro, $R J$ - Brazil. \\ Editorial related to the article: Lung Ultrasound as a Triage Tool in an Emergency Setting during the Covid-19 Outbreak: comparison with CT Findings
}

Since the first descriptions on the use of lung ultrasound (LUS) for bedside evaluation of critically ill patients, ${ }^{1}$ important publications have underscored the utility of LUS for the detection of a wide array of pulmonary and pleural diseases, as well as for the differential diagnosis of acute respiratory failure, ${ }^{2}$ proving to be an important tool for a point-of-care based examination and an extended resource for examining critical patients, cited by many as the "new stethoscope".,34

The coronavirus disease-2019 (COVID-19) outbreak has emerged as a global health burden since late 2019, challenging healthcare systems around the world due to the constant need for intensive care support and isolation of patients, many of whom evolve with severe hemodynamic involvement, leading to high morbidity and mortality. ${ }^{5}$ This critical scenario highlights the importance of appropriateness and best appliance of all diagnostic and therapeutic resources. In this regard, the use of point-of-care ultrasound (POCUS) is proving to be a cost-effective tool for the management of these potentially critical patients, from the initial presentation (triage) to in-hospital management and post-discharge follow-up 6 .

Chest computed tomography (CT) has a high sensitivity in the detection of findings compatible with COVID 19 pneumonia, ${ }^{7}$ and some scores derived from

\section{Keywords}

COVID-19/complications, Pandemics, Acute Failure Respiratory; Morbidity; Mortality; Pleural Diseases/ Diagnosis; Critic care; Ultrasonography/methods; Computed, Tomography.
CT-findings have a good correlation with short term outcomes, with a higher mortality in cases of a higher percentage of lobar involvement. ${ }^{8}$ Considering the fact that CT scans are not available in all emergency clinics, especially in underdeveloped countries or remote locations, and that, in a pandemic scenario, the sheer number of patients needing evaluation may surpass the actual capacity of the system, alternative diagnostic modalities are a good reinforcement to aid in patient evaluation and severity assessment.

This issue of the International Journal of Cardiovascular Sciences presents the results of an interesting study entitled "Lung Ultrasound as a Triage Tool in an Emergency Setting during the Covid-19 Outbreak: comparison with CT Findings" written by Alcantara et al. ${ }^{9}$ This study sheds new light on the discussion of this important topic, adding relevant information about the value of LUS for triage of suspected COVID-19 patients examined at the emergency clinic (EC), as compared to CT findings.

The authors performed LUS in 20 patients admitted to the EC with clinically suspected COVID-19 and registered the number of abnormal segments detected by LUS. Each lung segment was also scored based on the degree of severity of LUS findings and a final summed score was calculated for each patient. The number of affected segments and the summed score was then compared to standardized CT findings.

Interestingly, patients with a low LUS score $(\leq 1)$ or number of affected segments $(\leq 1)$ showed an excellent correlation with minor pulmonary involvement illustrated in the CT scan, thus correctly classifying these patients into a lower risk group. Conversely, more

Mailing Address: Filipe Carvalho

Avenida Ataulfo de Paiva, 669. Postal Code: 22640-102, Leblon, Rio de Janeiro, RJ - Brazil.

E-mail: filipepenna@me.com 
extensive lung abnormalities seen on LUS, defined as the presence of $\geq 3$ abnormal segments or a score $>7$ showed good sensitivity and excellent specificity $(81.8 \%$ and $100 \%$, respectively) in predicting extensive lung involvement via CT scans ( $\geq 6$ CT segments), with AUCs of 0.980 for a number of affected segments via LUS and a LUS score of 0.975 .

Characteristic LUS lesions suggestive of COVID-19 pneumonia have been described since the initial stages of the outbreak. ${ }^{10}$ The sensitivity of LUS in detecting a alveolo-intersticial pattern in COVID-19 patients was even greater than that detected by $\mathrm{CT}$, as shown in the findings from Yang et al., ${ }^{11}$ demonstrating the potential applicability of this technique to early diagnosis and treatment. A study conducted by Zieleskiewicz et al. ${ }^{12}$ showed an association of a high LUS score with the use of mechanical ventilation, and with a $\mathrm{SpO} 2 / \mathrm{FiO} 2$ ratio below 357, also indicating a prognostic value of LUS

\section{References}

1. Lichtenstein $D, A x l e r O$. Intensive use of general ultrasound in the intensive care unit. Intensive Care Med. 1993;19(6):353-5. doi:10.1007/BF01694712

2. Lichtenstein DA, Mezière GA. Relevance of Lung Ultrasound in the Diagnosis of Acute Respiratory Failure*: The BLUE Protocol. Chest. 2008;134(1):117-25. doi:10.1378/chest.07-2800

3. Narula J, Chandrashekhar Y, Braunwald E. Time to Add a Fifth Pillar to Bedside Physical Examination. JAMA Cardiol. 2018;3(4):346. doi:10.1001/ jamacardio.2018.0001

4. Buonsenso D, Pata D, Chiaretti A. COVID-19 outbreak: less stethoscope, more ultrasound. Lancet Respir Med. 2020;8(5):e27. doi:10.1016/S22132600(20)30120-

5. Rodriguez-Morales AJ, Cardona-Ospina JA, Gutiérrez-Ocampo E, et al. Clinical, laboratory and imaging features of COVID-19: A systematic review and meta-analysis. Travel Med Infect Dis. 2020;34:101623. doi:10.1016/j. tmaid.2020.101623

6. Gargani L, Soliman-Aboumarie H, Volpicelli G, Corradi F, Pastore MC, Cameli M. Why, when, and how to use lung ultrasound during the COVID-19 pandemic: enthusiasm and caution. Eur Hear J - Cardiovasc Imaging. 2020;21(9):941-8. doi:10.1093/ehjci/jeaa163

7. Bernheim A, Mei X, Huang M, Yang Y, Fayad ZA, Zhang N, et al, . Chest CT Findings in Coronavirus Disease-19 (COVID-19): Relationship to Duration of Infection. Radiology. 2020;295(3):200463. doi:10.1148/radiol.2020200463 findings. A greater extent of pulmonary involvement in the CT scan was correlated with severe COVID-19 presentations $(\mathrm{p}<0.0001)$, CRP levels $(\mathrm{p}<0.0001$, $\mathrm{r}=0.6204)$, and $\mathrm{D}$-dimer $(\mathrm{p}<0.0001, \mathrm{r}=0.6625)$. An increased risk of mortality was observed in patients with extensive disease, as shown via a CT scan, both in univariate (HR, 8.33; 95\% CI, 3.19-21.73; $\mathrm{p}<0.0001)$ and multivariate (HR, 3.74; 95\% CI, 1.10-12.77; $\mathrm{p}=0.0348)$ analyses. $^{8}$

Findings from Alcantara et al. ${ }^{9}$ adds new insight to this issue by demonstrating the potential power of LUS as a triage tool for COVID-19 in ECs, since LUS showed a good correlation with both minor and extensive pulmonary involvement, as defined via CT scans. Further studies with larger cohorts and external validation are still required to answer whether LUS as a triage tool translates into an improved workflow and a safe hospital discharge for patients in the EC setting.

8. Francone M, Iafrate F, Masci GM,Coco S, Cilia F, Manganaro L, et al. Chest CT score in COVID-19 patients: correlation with disease severity and short-term prognosis. Eur Radiol. July 2020. doi:10.1007/ s00330-020-07033-y

9. Alcantara ML De, Paulo M, Bernardo L, Lustosa RP, Tayah M, Chagas LA,et al. Lung Ultrasound as a Triage Tool in an Emergency Setting during the Covid-19 Outbreak : comparison with CT Findings. Int J Cardiovasc Sci. 2020; 33(5):479-487.

10. Buonsenso D, Piano A, Raffaelli F, Bonadia N, de Gaetano Donati K, Franceschi F. Point-of-Care Lung Ultrasound findings in novel coronavirus disease-19 pnemoniae: a case report and potential applications during COVID-19 outbreak. Eur Rev Med Pharmacol Sci. 2020;24(5):2776-80. doi:10.26355/eurrev_202003_20549

11. Yang Y, Huang Y, Gao F, Yuan L, Wang Z. Lung ultrasonography versus chest CT in COVID-19 pneumonia: a two-centered retrospective comparison study from China. Intensive Care Med. 2020;46(9):1761-3. doi:10.1007/s00134-020-06096-1

12. Zieleskiewicz L, Markarian T, Lopez A, Taguet C, Mohammed N, Boucekine $\mathrm{M}$, et al. Comparative study of lung ultrasound and chest computed tomography scan in the assessment of severity of confirmed COVID-19 pneumonia. Intensive Care Med. 2020;46(9):1707-13. doi:10.1007/s00134-020-06186-0 